American Journal of Pharmaceutical Education 2019; 83 (5) Article 6958.

\title{
BRIEF
}

\section{Exploring the Consequences on Memory of Students Who Know They Have Access to Recorded Lectures}

\author{
Bianka Patel, BS, Grace Yook, BS, Sarah Mislan, BS, Adam M. Persky, PhD \\ UNC Eshelman School of Pharmacy, University of North Carolina at Chapel Hill, North Carolina \\ Submitted January 19, 2018; accepted May 8, 2018; published June 2019.
}

\begin{abstract}
Objective. To elucidate how students' knowledge of future access to recorded lectures impacts their ability to immediately recall, to delay recall, and to restudy information.

Methods. Seventy-eight participants were randomly divided into two groups: knowledge of future access to recorded lectures after class and knowledge of no future access to recorded lectures after class. Participants viewed two mini lectures (10-12 minutes each) in a simulated classroom. Participants were told whether they would or would not be able to restudy lectures through future access to the recorded lectures just prior to their test one week later. Participants were tested immediately following the lectures and after a one-week delay. Prior to the delayed test, participants restudied one of the two lectures. The primary outcome was the participants' performance on the lecture material following immediate testing. Secondary outcomes included performance on delayed tests, performance after restudying the lectures and note-taking behavior.

Results. Having access to a recorded lecture did not influence immediate recall. One week after the simulated class, reviewing videos did improve performance $(\mathrm{d} \sim .70)$. Participants with knowledge of no future access forgot less information $(\mathrm{d}=.42)$ over time compared to the group that knew they had future access $(\mathrm{d}=.53)$; even though this latter group wrote longer notes.

Conclusion. These findings suggest that there is no mnemonic-benefit to having knowledge of access to recorded classes. Reviewing recorded lectures did improve scores on an immediate test. However, participants with knowledge they had access to the recorded lectures to restudy them had larger effect sizes for loss of material.
\end{abstract}

Keywords: recorded classes, desirable difficulties, note-taking, study strategies, memory

\section{INTRODUCTION}

An increasing number of health profession programs record lectures and grant students access to these lectures. This practice has potential advantages and disadvantages for classroom behavior and post-class study. Theoretically, if students can access a recorded lecture after class, they can minimize the amount of material they need to commit to memory because this recording serves to externalize memory. As a result, students can potentially reallocate that saved memory into deeper processing of a topic. In addition, students can review this externally stored information to re-study and not rely on their memory or their notes. The purpose of this pilot study is to examine if knowledge of access to and reviewing of recorded lectures has immediate benefits for those who attend lecture. This will be explored in a

Corresponding Author: Adam M. Persky, 2312 Kerr Hall, CB 7569, 301 Pharmacy Lane, UNC Eshelman School of Pharmacy, Chapel Hill, NC 27713. Tel: 919-966-9104.

E-mail: apersky@unc.edu simulated classroom environment to minimize confounding variables.

Recorded lectures are typical in many medical and pharmacy schools and these recordings are generally made available to students post-class. Students often know they have access to these videos for later use and use these recordings for a variety of reasons. One reason students favor access to recorded lectures is the perception of increased speed in acquiring knowledge. ${ }^{1}$ Another reason they prefer access to recorded lectures is to replace attending class with the recordings. ${ }^{2}$ Consequentially, medical schools and other health professional programs that release recorded lectures observe problems with attendance. ${ }^{3,4}$ Additionally, by knowing they have access to the recorded lectures after class, students may, theoretically, be able to learn more during class.

Imagine if students knew that notes from the lecture would be provided - then it is possible that students would feel less stress, and apprehension about note acquisition. 


\section{American Journal of Pharmaceutical Education 2019; 83 (5) Article 6958.}

They can then dedicate their energy on understanding the material throughout the lecture. This could ultimately result in a more holistic learning opportunity. This phenomenon has been referred to as the Efficient Encoding Hypothesis. ${ }^{5}$ That is, having access to complete notes or in the case of this study, recorded lectures - represents an externalization of information. By externalizing information, students can potentially focus more deeply on the discussed material rather than taking notes. The reduction in note taking can consequentially lessen a student's demands on their memory; thus, allowing them to focus more attention on the content. In this scenario, the reduction in cognitive demand may be beneficial and increase memory strength.

But the question arises, if students are not taking notes are they missing out on the learning process? The answer is, yes, potentially. This phenomenon would be consistent with the Desirable Difficulty Hypothesis, which suggests that learning requires a level of difficulty that can be overcome with effort. ${ }^{6}$ Since note taking is an important encoding and processing strategy, taking less notes or lower quality notes could subsequently lessen memory strength. ${ }^{7-9}$ The Efficient Encoding Hypothesis and Desirable Difficulty Hypothesis propose opposing outcomes for learning when students know there is access to post-lecture recordings. In this study, we investigated whether the knowledge of having access to recorded lectures affects students' learning both immediately and after a delay. We also investigated if there is an impact on note-taking behavior, as this may influence learning.

Regardless of the effect on memory and note taking, students desire recorded lectures so that they can review them prior to assessments or fill in any gaps in their notes. This may have learning benefits. However, if students are attending class and reviewing the recordings after class, this strategy may not be the optimal use of time. Reviewing recorded lectures after attending a lecture is similar to re-reading or reviewing material, which may not be the most efficient study strategy; rereading often leads to high immediate recall performance but a reduction in their delayed recall performance, which suggests that students are not deeply learning the material. ${ }^{10,11}$ While restudying the lectures does not tend to increase long-term retention, it increases the familiarity of the content and can make students feel that the content is well-learned, which can be problematic. ${ }^{12-14}$ Reviewing lectures for classes a student already attended also adds a significant portion of time spent studying. As such, in this study we investigated if performance improved immediately after restudying the videos and conducted an exploratory cost-effect analysis.
In summary, there are four main objectives to this pilot project. The first objective is to understand if immediate quiz performance is affected if students know they have future access to recorded lectures. The second objective addressed long-term learning performance if students know they have access to lecture recordings but do not review them. The third objective addressed if re-studying from videos improves performance of immediate recall. The fourth objective is related to the last two and addressed if studying from recorded lectures was worth the time and effort. Researchers asked a final, secondary question regarding the influence that note taking may have had on performance. These questions will be explored in a simulated classroom environment in which participants will be told whether they will have access to recorded lectures prior to being tested. We will examine immediate performance, delayed performance, note length, and performance after restudying the videos one week later.

\section{METHODS}

This study was part of a larger published study. ${ }^{15}$ Thus, participants and methods from this study were identical to the prior work but explained briefly here.

Participants were recruited from a school of pharmacy because the results would be directly related to school policies and course design. Participants were offered either extra credit in certain courses or a $\$ 25$ gift card for participating in the study. Participants were recruited from all professional classes. In general, each professional year has the same admissions criteria.

The study was a parallel arm study with two groups: students were instructed they will have access to the recorded lectures and could restudy them before their final assessment at one week (access group); and students were instructed they would not have access to or the ability to restudy the recorded lectures before their final assessment at one week (no access group). This study design consisted of six total phases and was a 2 (recording access: access or no access) x 2 (time: immediate testing or delayed testing) x 2 (restudy: no restudy or restudy) design (Figure 1). Knowledge of recording access was the only variable compared between subjects; all other variables were compared within subjects.

Participants were scheduled in small cohorts depending on availability (eg, five to 15 participants at once) to simulate a classroom environment. An "instructor" (ie, study investigator) was present at each session to provide overall instructions to the participants and manage the lecture videos.

In Phase I, study investigators instructed the participants they would watch two video lectures and to behave as if they were in a real classroom setting. Furthermore, 


\section{American Journal of Pharmaceutical Education 2019; 83 (5) Article 6958.}

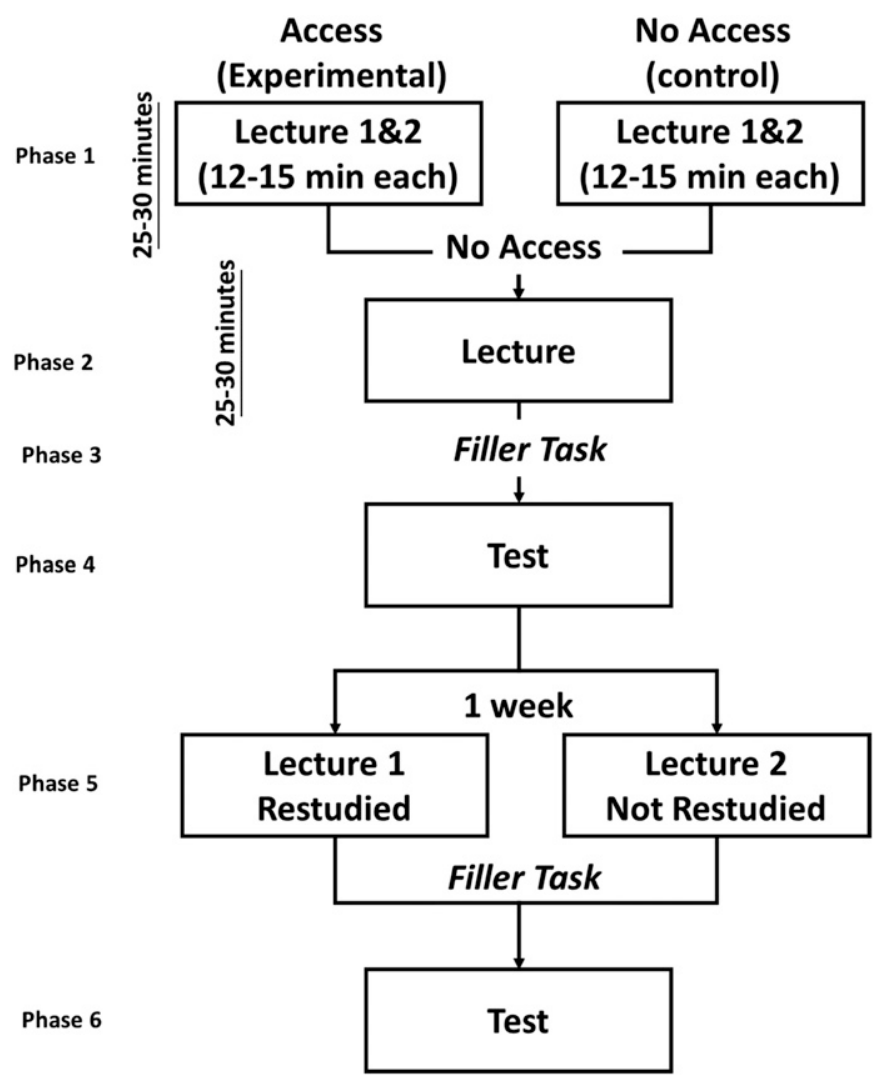

Figure 1. Diagram of Study Design. Participants were instructed whether they would have access or no access to recorded lectures to restudy them at one week. Participants were assessed immediately after lecture (Phase 4) and one week later (Phase 6) after restudying or not restudying the recorded lectures.

they were informed they would be tested on the material in one week. Half of the participants were instructed that just before the assessment one week later, they would review these same videos; the other half were told they would not have access to these videos for review. For each lecture, participants had blank paper and a pen for taking notes.

In Phase II, participants watched two more video lectures to simulate a 50 -minute class period total - these videos were not included in the current study. After students finished the final two videos, participants completed a filler task (visuospatial games) for approximately 10 minutes. Participants then completed a 40-question assessment on all four videos (described later). However, the primary outcome for this study was to evaluate test performance on the questions derived from the first two video lectures only.

One week after the first session, participants returned to "class." All participants were then randomized to review one of the two videos they had watched initially. After participants restudied the lecture content, they were given a filler task, just as they were earlier. Finally, the same assessment on all four videos was administered.

Traditional classroom lectures were emulated with publicly available video lectures. The video lectures were independent of pharmacy content to reduce the effect of students' prior knowledge on study outcomes. In addition, videos were used to reduce the variability of an instructor live lecturing multiple times across various content areas. Each video was 10-12 minutes in length and the content was related to astronomy (Crash Course on Astronomy series, YouTube), physics, or earth science. A total of eight videos were used in the study, and each participant viewed four of these lectures. The lectures watched were counterbalanced, randomized and blocked across conditions to reduce any content-related effects. That is, each day the study was conducted, participants received videos in a different order or different videos to remove any effects of the content difficulty (eg, easier or harder content areas).

Learning was assessed through a test on each video lecture. Each video was tested with a 10-question assessment containing a mixture of question types to parallel authentic examinations (six short-answer or fill in the blank, two multiple-choice, and two true/false). These questions were knowledge- or concept-based (ie, lower levels of Bloom's Cognitive Taxonomy) with single correct answers.

Students completed a filler task in between watching lectures and taking the test to keep them cognitively engaged, allowing for better assessment of long-term memory instead of immediate recall (ie, information in working memory). This material consisted of visuospatial brainteasers (eg, Sudoku) or math problems in paper-andpencil format.

The primary outcome was immediate recall performance. The secondary outcome was test performance during delayed recall (one week later) under the conditions of having the ability to restudy lectures. We conducted a $t$-test for treatment (access or no access), a paired $t$-test for time (immediate test vs delayed test) and a paired $t$-test for the restudy conditions (restudying videos vs not restudying). We assessed participants' notes by counting the number of words and diagrams from each student's notes. Pearson correlations were used for the associations between test performance and length of notes or number of diagrams. An additional secondary outcome was the cost-effect analysis with cost being the additional time to restudy the video and effect being the learning gains or deficits (delayed recall vs immediate recall).

The $p$ value was set at $p<.05$ except for three or more comparisons, for which it was adjusted using the Holm-Bonferroni correction (IBM Corp. Released 2013. IBM SPSS Statistics for Windows, Version 22.0. Armonk, 


\section{American Journal of Pharmaceutical Education 2019; 83 (5) Article 6958.}

NY: IBM Corp). This study was approved by the University of North Carolina Institutional Review Board.

\section{RESULTS}

Seventy-eight participants completed the study, with 39 participants in each group. Each group had a similar composition between first- and second-year students, but one group had more third-year students (zero vs six).

The first objective was to understand if quiz performance immediately after "lecture" is affected if students know they have future access to recorded lectures. During immediate recall, there was no significant difference on test performance between the access group $(\mathrm{M}=57 \%$, $\mathrm{SD}=14 \%)$ and no access group $(\mathrm{M}=55 \%, \mathrm{SD}=17 \%)$ $(\mathrm{d}=.13, p=.45)$. This data suggests that there was neither a benefit nor a detriment for participants if they knew they would have future access to the recordings on immediate recall.

The second objective addressed if long-term retention is affected if students know they have access to lecture recordings but did not restudy material. Both the access and no access groups had significant decreases in performance over time (access: $\mathrm{d}=.53, p<.001$; no access $=.42, p<.001)$ but there was no difference in scores between the access $(\mathrm{M}=48 \%, \mathrm{SD}=24 \%)$ and no access $(\mathrm{M}=46 \%, \mathrm{SD}=22 \%)$ conditions $(p=.73)$. However, based on effect size and $95 \%$ confidence intervals of forgetting over time, there is some indication that the no access group lost less knowledge over time compared to the access group (Figure 2). That is, forgetting occurred under all conditions but may be more pronounced under the access condition.

The third objective addressed was whether restudying videos improved performance. Participants who restudied from the video had higher performance than when they did not restudy the video; this was true for the access $(\mathrm{d}=.72, p<.001)$ and no access condition $(\mathrm{d}=.73$, $p<.001)$. There was no difference in test performance between conditions ( $M=64 \%$ vs $63 \%, p=.80)$. When examining the gains from a baseline, the data showed that the access group and no access group had similar gains in test performance after reviewing the video (Figure 2). Compared to not restudying the videos, rewatching the videos improves the immediate recall performance.

We explored the learning benefit from restudying the video considering the amount of time it would take to restudy the lectures. Reviewing a lecture doubled the time on a task (ie, attend a lecture and then re-watch the lecture) and this process yielded an approximate $25 \% \mathrm{im}$ provement on short-term recall compared to not reviewing the lectures. To state it another way, restudying the videos offset a student's memory lapses under immediate recall

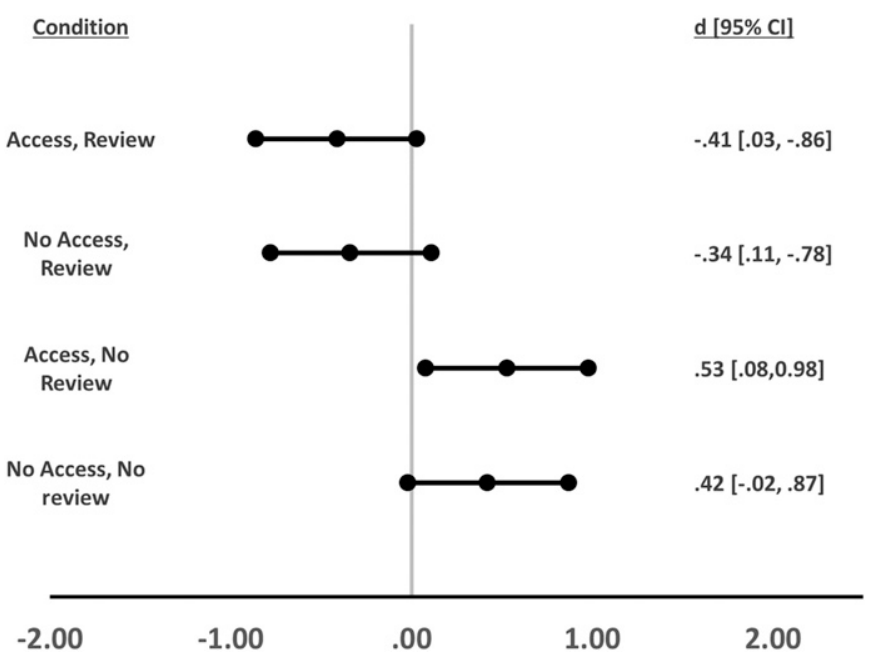

Figure 2. Impact of Retention Time (10 minutes or 1 week) and Review (review vs no review). Confidence intervals for the effect sizes related to the time effects - immediate recall (10 minute) vs delayed recall (1 week) with negative effect sizes indicating gain in performance.

conditions, but this came with a larger increase in time allocation.

Since the process of note taking may affect the retention of knowledge (ie, the "desirable difficulties hypothesis), the final question asked was whether note taking behavior was associated with test performance. We examined the relationship between scores during immediate and delayed recall tests in relationship to participants' notes. Participants' access, or lack thereof, to the recorded lecture dictated both the length of notes and the number of participants that took notes (Table 1). The access group wrote longer notes and made more diagrams when compared to the no access group.

Correlation analysis revealed a condition by note interaction and that making diagrams positively predicted test performance under all conditions (Table 1). Participants with no access had a positive correlation between note length and performance at immediate $(\mathrm{d}=1.2)$ and delayed $(\mathrm{d}=.69)$ recall when videos were not reviewed. In other words, when participants were told they would not have access to recordings, taking notes and creating drawings/diagrams was associated with increased performance. Conversely, the participants with access took longer notes but this did not correlate with performance.

\section{DISCUSSION}

This study is one of the first to investigate the impact of knowledge of future access to recorded lectures on recall of information. When participants attended a simulated lecture and were told they would have access to the recorded lecture and could restudy them, there was no 


\section{American Journal of Pharmaceutical Education 2019; 83 (5) Article 6958.}

Table 1. Summary of Note Quality Based on Access to Recorded Videos and Relationship to Quiz Performance

\begin{tabular}{|c|c|c|c|c|c|c|}
\hline \multirow[b]{3}{*}{ Count } & \multirow{2}{*}{\multicolumn{2}{|c|}{ Immediate Recall }} & \multicolumn{4}{|c|}{ Delayed Recall } \\
\hline & & & \multicolumn{2}{|c|}{ Reviewed } & \multicolumn{2}{|c|}{ Not Reviewed } \\
\hline & Access & No Access & Access & No Access & Access & No Access \\
\hline Participants w/ Notes & $39(100 \%)^{a}$ & $21(54 \%)$ & - & - & - & - \\
\hline Participants w/ Diagrams & $16(41 \%)$ & $10(26 \%)$ & - & - & - & - \\
\hline Word Count (SD) & $98(44)^{a}$ & $35(47)$ & - & - & - & - \\
\hline Diagram Count (SD) & $0.9(1.3)^{\mathrm{a}}$ & $0.4(.9)$ & - & - & - & - \\
\hline Note to Quiz Correlation & $\mathrm{r}=.076$ & $\mathrm{r}=.51^{\mathrm{b}}$ & $\mathrm{r}=.032$ & $\mathrm{r}=.12$ & $\mathrm{r}=.16$ & $\mathrm{r}=.33^{\mathrm{b}}$ \\
\hline Diagram to Quiz Correlation & $\mathrm{r} .=43^{\mathrm{b}}$ & $\mathrm{r}=.44^{\mathrm{b}}$ & $\mathrm{r}=.43^{\mathrm{b}}$ & $\mathrm{r}=.29$ & $\mathrm{r}=.46^{\mathrm{b}}$ & $\mathrm{r}=.26$ \\
\hline
\end{tabular}

Access: participants who knew they had future access to recorded lectures to restudy them. no access: participants who knew they did not have future access to recorded lectures. Immediate recall: assessment soon after lecture (10 minutes). Delayed Recall: assessment 1 week after lecture. Reviewed: participants restudied the recorded lectures. Not reviewed: participants did not restudy the recorded lecture

${ }^{\mathrm{a}} p<.05$ vs access ( $t$-test or Chi-square)

${ }^{b} p<.05$ for the correlation

significant effect on immediate performance on that material. However, based on long-term retention and extrapolating to a classroom setting, the findings may suggest not only a lack of benefit, but a potential detrimental effect of learners knowing they have access to recorded class sessions in a lecture-based environment. However, there was some benefit to re-watching the lecture prior to a future assessment.

When students had a chance to review the video before their final assessment, there was a statistical difference in the performance on the reviewed content when compared to the un-reviewed content. This improvement is one reason medical students state that they review lectures: they can reinforce concepts and fill in gaps in their comprehension. ${ }^{1,2}$ Although re-watching videos improved performance, further research is necessary to determine if re-watching lectures is an optimal use of study time in comparison to restudying from notes or taking a practice test. Based on a cost-effect analysis, spending time to re-watch an entire lecture over again resulted in increasing performance by $25 \%$ compared to no restudy; other study strategies can be compared to re-watching lectures by considering time investment and outcome, especially for measures of long-term retention.

Despite the improvement seen from reviewing the video lecture, there may be some differences in the magnitude of effect of re-watching videos under the experimental conditions tested. Students who knew there was future access may have benefited more from reviewing the video, but this may be a function of the fact that this group forgot more information. This finding may support the idea that students who knew there was no future access to material had a higher mental investment at the initial learning phase. Taken together, these findings suggest some effect, albeit small, on the knowledge of having future access to videos. From an instructional standpoint, the results could mean that knowing there is future access to recorded lectures may lead to higher performance initially, but it also results in a reduction in knowledge retention. Thus, it may be advisable to inform students there is no future access to recordings.

Participant performance did not differ between the access and no access groups during immediate or delayed recall. However, the data indicated that the access group had larger effect sizes for material loss. This finding suggests that when participants knew they would have future access, they engaged in weaker encoding during lecture (ie, the learning phase) despite the higher quantities of note taking. These effects were small, but they may support the Desirable Difficulty hypothesis. That is, note taking may be an important part of the learning process.

Participants who had diagrams in their notes performed better overall, which potentially represents deeper processing and further supports the Desirable Difficulty Hypothesis. This was especially impactful for the participants who were informed that they would not have access to recorded lectures. It was unexpected that participants within the access group took notes and, those that did take notes, took longer ones. There was a note taking by condition interaction with the no access group writing less notes. The rationale for this is unclear, but it may support the Efficient Encoding Hypothesis. Participants may have stronger encoding when not dividing their attention between note taking and paying attention to the lecture. Under the no access condition, participants took less notes, performed similarly to the access group, but they may retain more information over time. Ultimately, the findings of this study propose that, although competing, these hypotheses may be both explanatory and the prevailing hypothesis may be conditional on other factors. Taking notes may be an important mnemonic strategy as long as it does not divide attention. 


\section{American Journal of Pharmaceutical Education 2019; 83 (5) Article 6958.}

As this was one of the first investigations on the topic, there were some limitations and strengths. One of the strengths of this study is that it assessed the impact of knowledge of access to recorded lectures by using a simulated classroom environment with novel lecture content, which allows researchers to maintain control of both external factors and prior knowledge. Another strength is that learning was assessed both immediately and after a one-week delay. While interventions like cramming or re-reading, may result in high immediate performance, they may not yield higher long-term retention. Ultimately, as instructors, we care about the long-term effects of the instructional environment. A potential downside is that a simulated lecture style classroom may not accurately represent the true nature of a professional curriculum: face-to-face instruction, active learning, longer class periods, topics related to their discipline or the repeated demands on memory from multiple courses. In addition, testing occurred relatively soon after students restudied the recorded lecture, which may not accurately reflect true restudy conditions. However, some of the trends and findings of this study do reflect what has been observed in the classroom. For example, the knowledge that access to recorded lectures resulted in poorer performance on long-term retention is consistent with classroom studies that found students who access recordings more frequently perform poorer on examinations. ${ }^{12}$

The impact of recorded lectures on learning needs to be studied further both from an in-class learning perspective (eg, do students learn more?) to their use of recordings after class (eg, are there better study strategies?). Some of these questions may be difficult to study in authentic classroom environments as there may be many confounding variables (eg, note taking, class attendance, interest, content, re-watching speed). Ultimately, the release of course recordings may depend on the nature of the course, student characteristics, and the goals of instruction, but research is needed to clarify best practices.

\section{CONCLUSION}

Informing students that they will have access to recorded lectures after the class is completed did not appear to have any beneficial effects on memory. There is some indication that students knowing they will not have access to recorded lectures resulted in stronger memory formation with less deterioration in knowledge over time, but these effects are small. Finally, re-watching lectures does improve imminent examination performance, but it is unclear if other study methods would be more helpful or more cost-effective.

\section{REFERENCES}

1. Cardall S, Krupat E, Ulrich M. Live lecture versus video-recorded lecture: are students voting with their feet? Acad Med.

2008;83(12):1174-1178.

2. Gupta A, Saks NS. Exploring medical student decisions regarding attending live lectures and using recorded lectures. Med Teach. 2013;35(9):767-771.

3. Johnston ANB, Massa H, Burne THJ. Digital lecture recording: a cautionary tale. Nurse Educ Pract 2013;13(1):40-47.

4. Persky AM, Kirwin JL, Marasco CJ, May DB, Skomo ML, Kennedy KB. Classroom attendance: factors and perceptions of students and faculty in US schools of pharmacy. Curr Pharm Teach Learn. 2014;6(1):1-9.

5. Storm BC, Stone SM. Saving-Enhanced memory: the benefits of saving on the learning and remembering of new information. Psychol Sci. 2015;26(2):182-188.

6. Bjork EL, Bjork RA. Making things hard on yourself, but in a good way: creating desirable difficulties to enhance learning. In: Gernsbacher MA, ed. Psychology and the Real World: Essays Illustrating Fundamental Contributions to Society. New York, NY: Worth Publishers; 2011:59-68.

7. Benton SL, Kiewra KA, Whitfill JM, Dennison R. Encoding and external-storage effects on writing processes. J Educ Psychol. 1993;85(2):267-280.

8. Kiewra KA. A review of note-taking: the encoding storage paradigm and beyond. Educ Psychol Rev. 1989;1(2):147-172.

9. Kobayashi K. What limits the encoding effect of note-taking? a meta-analytic examination. Contemp Educ Psychol. 2005;30(2):242262.

10. Austin SDM. A study in logical memory. Am J Psychol. 1921;32(3):370-403.

11. Glover JA. The "testing" phenomenon: not gone but nearly forgotten. J Educ Psychol. 1989;81(3):392-399.

12. Dunlosky J, Rawson KA. Overconfidence produces underachievement: inaccurate self evaluations undermine students' learning and retention. Learn Instruct. 2012;22(4):271-280.

13. Kornell N, Rhodes MG, Castel AD, Tauber SK. The ease-ofprocessing heuristic and the stability bias: dissociating memory, memory beliefs, and memory judgments. Psychol Sci. 2011;22(6):787-794.

14. Little JL, McDaniel MA. Metamemory monitoring and control following retrieval practice for text. Mem Cogn. 2015;43(1):85-98. 15. Patel B, Mislan S, Yook G, Persky AM. Recorded lectures as a source of cognitiv off-loading. Am J Pharm Educ. In press; ajpe6793. 